(c) 2015. This manuscript version is made available under the CC-BY-NC-ND 4.0

\title{
Editorial
}

\section{Understanding the psychology behind performance-enhancement by doping}

\section{Andrea Petróczi \& Bernd Strauss}

After two years of consultation, the WADA Foundation Board Meeting accepted the revised Anti-Doping Code in Johannesburg in November 2013. This Special Section of Psychology of Sport and Exercise coincides with the new World Anti-Doping Code coming to effect in January 2015 and presents eight articles on doping in sport. Just as the World Anti-Doping Code versions in 2004, 2009 and now version 3 for 2015 and onwards signify a new era in anti-doping, research into understanding the psychology behind doping has progressed over the past 10 years. Collections of sport hoaxes almost always include the Ben Johnson's positive test for stanozolol (a synthetic anabolic steroid) after the $100 \mathrm{~m}$ final in the 1988 Seoul Olympics. His victory and world record, retrospectively both labelled as doping fuelled achievement, stands right next to Onischenko's cleverly rigged fencing sword in the 1976 Montreal Olympics, Maradona's hand in the 1986 Football World Cup final, the Spanish Paralympic gold medal in men's basketball in Sydney 2000 for a team of mostly healthy, not forgetting the several inventive ways for shortcuts in long distance races and manipulated boxing gloves. Upon closer examination however, doping stands out from the colourful history of sport cheats in many ways. First of all, doping does not guarantee winning or new records. Doping does not replace work, training and skills. Doping only expands the fix capacities of human performance. Doping is done to the self to enhance fixed capacities of performance, not to others. One athlete's use of doping does not prevent others doing the same - although many would argue that one's act puts undue pressure on others to follow suit. In fact, when doping is perceived to be widespread, it is often justified as an effort to level the playing field, not gaining advantage. Further complications arise from considerable inter-individual difference is both genetic make-up and lifestyle, both of which have impact on benefitting from and the detection of doping substances. Finally, the term doping uniquely refers to the prohibited and detested end-scale of the range of otherwise acceptable performance-enhancing practices.

Doping is one of the most controversial issues in sport psychology. Despite that performance-enhancing substances and methods have been used for centuries in various competitive situations, doping has become the conundrum of the 20th century competitive sport, and a very complex phenomenon. The absence of information on others' behaviour or the lack of co-operation leads to speculation about who is on doping and a distorted 
normative belief that doping is rampant in competitive sport - and beyond. Owing to the clandestine nature of the activity, the composite effect of self-interest actions decided upon in isolation can then easily lead to a collectively disadvantageous or irrational situation where doping use escalates.

At the individual level, motivation for using prohibited substances, like any other behavioural choice, stems from weighing negative and positive outcomes, including the chance of being detected and its consequences. This motivation leads to behavioural intention about doping and, in favourable situational contexts, to execution leading to use of or refrain from doping. Social cognitive models recognise that setting performance related goals and laying behavioural paths for actions to pursue these goals require self-regulatory efforts under some rational control. In the midst of conflicting expectations, blurred lines, analytical and pharmacological advances, 'war on drugs' mentality and increasingly common human enhancement practices outside sport, it is hard to see doping what it is: a way to enhance performance enhancement. Blurred lines exist between many aspects of doping, including: legality/illegality; acceptable/unacceptable; equity/equality; and more importantly between goals and behaviour. Anti-doping efforts failing to address the key motivators of doping in meaningful and practical ways are deemed to be ineffective. Effective anti-doping strategies must build on evidence-based understanding of how athletes see doping.

Literature seeking solutions for, or aiming to advance understanding of this problem has burgeoned in the last decade, with a significantly increased interest in Olympic years. Yet, owing to the complexity of external and internal factors contributing to doping, many intriguing questions have remained unanswered. Over the years it became evident that athletes' decision about doping cannot be explained by their permissive attitude alone but rather, the decision to dope is influenced by a host of other factors. Moreover, declared attitudes can be antecedents but also the results of doping behaviour - formed retrospectively based on experiences and feedback.

In the early 1900s, systematic training, which aimed at maximising human capacities was frowned upon and considered as non-gentlemanly behaviour. In the late 1900, although originally triggered by health concerns - pharmacological training is deemed unacceptable and unfair. Indisputably, doping is against the voluntarily accepted rules thus is cheating. However, from the behavioural point of view, doping can equally be a contextualised behaviour that is justified on the grounds of functionality. This functional view moves the decision regarding doping away from morality and fair play to being an effective way to increase athletic performance. In this process, doping is one step too far on the well-worn 
path of assisted performance enhancements comprises of accepted and widely used performance enhancing practices that, among other techniques, include a wide range of acceptable substances. The doping dilemma, as we have it today, is the unfortunate, and most likely unplanned consequence of conflicting normative expectations. On one hand, we want to see athletes going higher, faster and stronger than ever before. On the other hand, for the sake of health and fairness, the society feels compelled to control and place limits on the means by which athletic performance can be enhanced.

Chemically assisted human enhancement is an emerging phenomenon that characterises the later part of the 20th century; and what will continue to grow. It is not limited to doping in sport, but manifests in functional drug use to enhance human experience; non-medical use of cognitive enhancers; fat-burners and diet pills, cosmetic surgery and the use of doping substances (growth hormone, steroids) by general population. The intriguing question then is how can we justify the 'war against doping' in the era of human enhancements? What sets doping apart from all other forms of acceptable and cherished performance enhancements such as technological advancements in equipment or apparel; training methods, functional foods and the booming industry of dietary supplements? Can we righteously demand that athletes' $10-20$ years of investment into perfecting their techniques and physical and mental states should be done for just joy and fun?

The next phase is a journey which we need to start by stepping back and critically reflect upon what we have learnt from the past 10-15 years of social science research into doping. Questioning assumptions and conclusions we made in the past does not, by any means, implies that we got it wrong and now we have to set it right. There is no doubt that past assumptions can be defended and justified, but it is the future we should be concerned about, not the past. Along the way, we should resist the temptation of only generating outcomes that have direct relevance to anti-doping. Doping research to date is characterised by studies that are often encompass new methods and its applications within a single design. Blinded by the desire for finding directly relevant outcomes and being relatively new 'discipline', doping research has suffered from the unfortunate trend that not enough attention is given to theoretical and methodological aspects. On a long run, we would make bigger impact and play an influential part in advancing doping research with embracing blue-sky thinking and embarking on basic research more often. In this, we need to challenge the limitations and faulty assumptions - even if it means questioning ourselves in the process.

The collection of articles we proudly present in this Special Section seeks to communicate current thinking on doping behaviour and to advance our theoretical and 
empirical understanding of the individual level factors that encourages the use of or provide protection against doping. This Special Section brings together a collection of papers that presents a range of perspectives on performance enhancement by doping and provides a platform for sharing knowledge and critical examination of the current understanding of the factors relate to doping, with the view of informing preventive measures. The selected papers represent a wide range of topics, employ different methodologies and cut across social science disciplines.

Denis Hauw and Michael McNamee make an important contribution to grounding investigation into doping in specific research traditions. In their paper, they contrast and compare three research models, namely the cognitive, the 'drive' and the situated-dynamic approaches. They make a persuasive argument that these respective research frameworks made nuanced but important difference to how doping behavior is conceptualized and offer insights into the methodological aspects of research characterizes these research traditions.

Kelsey Erickson, Jim McKenna and Susan Backhouse use qualitative method to explore static and dynamic protective factors against doping. Their findings offer insights into the complex interaction between risk and protective factors at individual, psychosocial and societal levels but also highlights a concerning account of lack of awareness and knowledge. As a consequence, the authors suggest that anti-doping should move beyond the individual athlete and consider the contextual factors beyond the athlete's control that have potential impact on behavioural choices.

Staying within the qualitative research paradigm, Denis Hauw and Sara Mohamed explore consumption of doping substances through a dynamic and situated activity framework during the sporting life-course. Although the investigation relies on retrospective recollection of athletes' accessible feelings about substances over the course of their sport career, it offers a much needed contribution to empirical doping research that moves beyond a snapshot account of a single time-point. The authors posit that the parsimonious account of doping as a response to human capacities and/or as an intention to enhance performance limits our understanding of the doping phenomenon. The outcomes from this study offer support to the observation that contextual parameters, past and present, do play an important role in how doping is viewed by athletes.

Much of doping research to date has been conducted based on or used variables from the Theory of Planned Behaviour (TPB). The study by Derwin King-Chung Chan, Sarah Hardcastle, James Dimmock, Vanessa Lentillon-Kaestner, Robert Donovan, Matthew Burgin and Martin Hagger breaks the mould by using the model to test direct and indirect 
contributors of doping avoidance, not engagement. Their work also expands on the TPB model and explores modal salient behavioural, normative, and control beliefs as predictors for the directly measured TPB variables in four hypothesised expectancy-value models. Results of this study underscore the indirect influence of belief-based components on doping avoidance.

Vassilis Barkoukis, Lambros Lazuras and Peter Harris bring a new approach to anti-doping preventive intervention by offering evidence for the role of self-affirmation in the decision process about doping use. For the first time, the study recognizes that the high-risk group of doping user athletes may resist anti-doping messages that are in conflict with their behavior, but self-affirmation manipulation can help to reduce defensive cognitive processing of personally relevant and persuasive messages about changing behaviour.

\section{Philip Sullivan, Deborah Feltz, Kaitlyn LaForge MacKenzie and Seunghyun}

Hwang make their contribution to doping research on two accounts simultaneously when they focus on a less-prominent stakeholder group (coaches) and equip researchers with a tool to assess coaches' self-confidence in dealing with athletes suspected of doping. The paper provides by-the-book details and information for the readers on the reliability and validity of this new assessment tool aptly named 'Doping Confrontation Efficacy Scale'. To facilitate future applications, the authors kindly provide the full item set of their new scale in the Appendix of their article.

\section{Valentino Zurloni, Barbara Diana, Cesare Cavalera, Luca Argenton,} Massimiliano Elia and Fabrizia Mantovani add a unique flavour to doping research with their analysis of Lance Armstrong non-verbal behaviour accompanied his responses during the Oprah interview in 2013 about his doping use and conducts regarding other athletes. In addition to the intriguing findings, this paper also makes novel contribution to doping research by introducing a new methodology, T-pattern analysis, to detect nonverbal cues of deception.

\section{Stephen Moston, Terry Engelberg and James Skinner address a vital} developmental stage when investigates the perceived doping prevalence among adolescent athletes and non-athletes. Whilst the 'chicken or egg' dilemma (i.e., whether involvement in doping manifests in higher perceived prevalence or the perceived high prevalence acts as normative motivation) remains unresolved without a longitudinal enquiry, the article accentuates the utility of social projection in doping research. 
This captivating collection complements work on doping that previously appeared over the past 5 years in Psychology of Sport and Exercise which provided a scale for measuring declared doping attitudes (Petroczi \& Aidman, 2009), addressed methodological issues associated with self-reports on doping (Gucciardi, Jalleh \& Donovan, 2011; James, Nepusz, Naughton \& Petroczi, 2013), employed response-time based measures (Brand, Meltzer \& Hagemann, 2011; Schirlin, Rey, Jouvent, Dubal, Komano, Perez-Diaz, et al., 2009) and presented doping behavioural models (Barkoukis, Lazuras, Tsorbatzoudis \& Rodafinos, 2011; Johnson, 2012; Zelli, Mallia \& Lucidi, 2010).

Matching exactly in numbers, the articles presented in the Special Section (and the already published papers) are worthy companions. It is notable that whilst these individually make important contribution to our understanding of doping and preventive efforts, collectively they showcase the current trend in doping social science research and, without exception, set research agenda for the future.

We hope that the articles will encourage further cross-disciplinary collaboration in doping research and taken together, they will facilitate fertilisation of ideas between sport psychologists and researchers rooted in different scientific traditions.

Andrea Petróczi, Guest Editor*

School of Life Sciences, Kingston University London

Penrhyn Road, Kingston-upon Thames, KT1 2EE, UK

Bernd Strauss, Editor-in-Chief and Guest Editor

Institute of Sport and Exercise Sciences, Department of Sport Psychology, University of Muenster

Horstmarer Landweg 62b, 48149 Muenster, Germany

* Corresponding author

Tel.: 44 (0)20 84172436

E-mail address: a.petroczi@kingston.ac.uk

\section{References}


Barkoukis, B., Lazuras, L., Tsorbatzoudis, H., \& Rodafinos, A. (2011). Motivational and sportspersonship profiles of elite athletes in relation to doping behaviour. Psychology of Sport and Exercise, 12(3), 205-212. DOI: 10.1016/j.psychsport.2010.10.003

Brand, R. Melzer, M., \& Hagemann, N. (2011). Towards an implicit association test (IAT) for measuring doping attitudes in sports. Data-based recommendations developed from two recently published tests. Psychology of Sport and Exercise, 12(3), 250-256. DOI: 10.1016/j.psychsport.2011.01.002

Gucciardi, D.F., Jalleh, G., Donovan, R. (2010). Does social desirability influence the relationship between doping attitudes and doping susceptibility in athletes?, Psychology of Sport and Exercise, 11(6), 479-486. DOI: 10.1016/j.psychsport.2010.06.002

James, R.A., Nepusz, T., Naughton, D.P., \& Petróczi, A. (2013). A potential inflating effect in estimation models: Cautionary evidence from comparing performance enhancing drug and herbal hormonal supplement use estimates. Psychology of Sport and Exercise, 14(1), 84-96. DOI: 10.1016/j.psychsport.2012.08.00

Johnson, M.B. (2012). A systemic social-cognitive perspective on doping. Psychology of Sport and Exercise, 13(3), 317-323. DOI: 10.1016/j.psychsport.2011.12.007

Petróczi, A., \& Aidman, E. (2009). Measuring explicit attitude toward doping: Review of the psychometric properties of the Performance Enhancement Attitude Scale, Psychology of Sport and Exercise, 10(3), 390-396. DOI: 10.1016/j.psychsport.2008.11.001

Schirlin, O. Rey, G., Jouvent, R., Dubal, S., Komano, O., Perez-Diaz, F., \& Soussignan, R. (2009). Attentional bias for doping words and its relation with physical self-esteem in young adolescents. Psychology of Sport and Exercise, 10(6), 615-620. DOI: 10.1016/j.psychsport.2009.03.010

Zelli, A., Mallia, L., Lucidi, F. (2010). The contribution of interpersonal appraisals to a social-cognitive analysis of adolescents' doping use. Psychology of Sport and Exercise, 11(4), 304-311. DOI: 10.1016/j.psychsport.2010.02.008 